\title{
Powering over single mode fiber by shortening working time of loT device
}

\author{
Edvin Skaljo ${ }^{1 *}$, Aljo Mujcic ${ }^{2}$, Petr Munster ${ }^{3}$, Anis Maslo ${ }^{4}$, Mujo Hodzic ${ }^{5}$, Muhamed \\ Duhandzic $^{6}$ and Emir Karamemedovic ${ }^{7}$ \\ ${ }^{1}$ Department for Physics, University of Sarajevo, Zmaja od Bosne 33, Bosnia and Herzegovina \\ ${ }^{2}$ Faculty of Electrical Engineering, University of Tuzla, Bosnia and Herzegovina \\ ${ }^{3}$ Dept. Telecommunications, Brno University of Technology, Brno, Czech Republic \\ ${ }^{4}$ Deparment for investment, BH Telecom Sarajevo, Bosnia and Herzegovina \\ ${ }^{5}$ Polytechnic Faculty, University of Zenica, Bosnia and Herzegovina \\ ${ }^{6}$ Medical school Zenica, Bosnia and Herzegovina \\ ${ }^{7}$ FabLab Sarajevo, Zmaja od Bosne 8, Bosnia and Herzegovina
}

\begin{abstract}
Fiber optics has revolutionized telecommunication with its superior bandwidth and distance it can span. For its use in IoT networks, some of the limiting factors are the high cost of new installations and the need to power the end device by electrical current. The installations are a part of long-term investments, and one can expect this to be an ever-smaller issue as more fibers are installed. Typically, the newly installed cables contain single-mode fibers. There are a lot of reports on transport of power over fiber, however, majority recommend using multi-mode fibers with a large core or double-clad fibers. In our approach, instead of increasing the core of the fiber, we focus on the possibility of shortening the working time of IoT devices, using the existing single-mode fiber for powering. Also, instead of an expensive PV (photo voltaic) cell with small dimensions and a high efficiency, we propose using the commercially available larger PV cells with an air gap between the end of the fiber and the cells. In accordance with our approach, we successfully conducted an experiment.
\end{abstract}

\section{Introduction}

Internet of Things (IoT) networks of devices are growing daily and changing our environment. According to the forecast of the company Statista, the number of IoT devices is expected to triple in the next 10 years (from 8.75 billion in 2020 to 25.4 billion in 2030) [1]. Many IoT devices are sensors that usually consume low power as they typically do not need to be awake all of the time. In sleep-mode, sensors draw from 0 to several $\mu \mathrm{A}$. While awake, the electrical current can go from a few to several hundred of $\mathrm{mA}$. The awake mode can be from one time per several milliseconds to one per day or so. Duration of the awake mode is typically less than 1s [2]. For example, temperature for weather conditions should be sent every hour with a duration of the awake mode of less than $1 \mathrm{~s}$. It should be mentioned that, the longer the sleep mode, the longer is the lifetime of the sensor.

${ }^{*}$ Corresponding author : skaljo@hotmail.com; edvin.skaljo@pmf.unsa.ba 
IoT sensors are often connected to the Internet by LoRa, 3/4/5 G, NB-IoT, Sigfox etc. Fiber optics is rarely used due to two main reasons, namely cost and powering. The cost of fiber optics is very high if the system requires new installations. If fiber optics networks are already installed, the share of fiber optics in the cost of the complete IoT network is significantly reduced. Currently a lot of fiber optic cables are installed underground or on poles. Most of them are based on the standard single-mode fiber (SSMF) or some compatible type, for example FTTH networks are based on SSMF and smaller parts on fibers according to ITU Recommendation G.657.

The other drawback, powering over fiber, has been intensively studied. These studies are characterized by proposing use of multimode fibers or other types of fiber with larger core diameter with possible use of batteries as an auxiliary power supply [3][4][5].

\section{Powering over Fiber}

There are two basic power transmission architectures over fiber optics depicted in Fig. 1:

- Separating fibers for energy transmission and information transmission;

- Common fiber for powering and information.
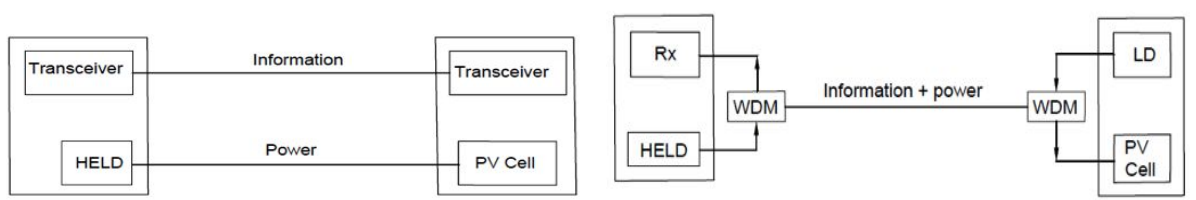

Fig. 1. Power transmmision arcihtecture: (left) two separate fibers for power transmission (right) Common fiber for powering and information flow.

On the sensor side, PV (Photo Voltaic) cell can be used in the following three ways:

1. directly (Fig. 2. a).

2. parallel to a battery (Fig. 2. b);

3. charging a capacitor as the energy storage (Fig. 2.c);

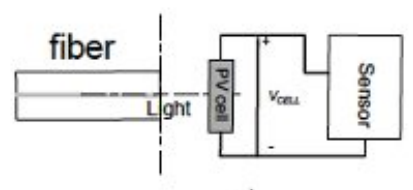

a)

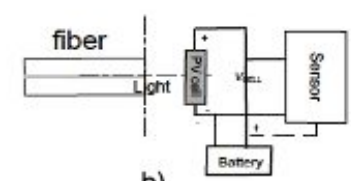

b)

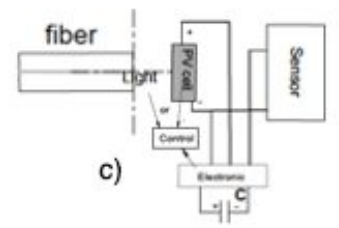

Fig 2. Three cases of receiver: a) Direct mode of PV Cell usage; b) Powering over battery; c) Charging the capacitor

The first case is the simplest but also the most impractical for long-distance applications. It is difficult to achieve sufficient energy for the state of wakefulness at a distance of more than a few hundred meters. In the second case, the transferred energy can be stored in the battery during the sleep mode and consumed during the awake mode. The disadvantage of this model is the need to have a battery, and occasionally replace the battery every few years. The third model has a longer life without intervention because the capacitor lasts significantly 
longer than a battery. However, the current has a variable intensity, so electronic devices must be used to regulate the voltage or current.

\subsection{Powering over Fiber - our approach}

In our approach, we propose to use power transmission over the same fiber through which the information is sent, according to Fig. 1. right, and the receiver side in according to Fig. 2.

The more detailed approach:

- SSM fiber because it is all around us and has a very low attenuation. This allows the development of cost-effective devices for long-distance powering of sensors.

- Simple PV cells commercially available, where instead of high field concentrations and high efficiency. PV cells have to be small in dimension with high efficiency to provide sufficient voltage to power sensors from a small surface. In [4], a receiver with 4 diodes in series in a circle with a diameter of $1.5 \mathrm{~mm}$ is proposed to achieve a voltage level above $2 \mathrm{~V}$. High efficiency and nonstandard PV cells can cost several times more than the complete sensor.

- A capacitor for energy storage. A battery can be used as an energy accumulator, but its lifetime is limited. The lifespan of fiber optics is 25 years or more, electronics as well, so the use of a capacitor is recommended instead of the battery because there is no need for any maintenance for many years of exploitation. The disadvantage of using a capacitor instead of a battery is the variable power supply, namely the capacitor during discharge gives a voltage that decreases exponentially, which requires additional voltage stabilization.

- $\quad$ The distance between the end of fiber and PV cells is used to increase the voltage from PV Cell to charge a capacitor. In [6] MPPT (Maximum Power Point Tracking) was used to increase the capacitor charging voltage. In our approach we easily obtain a voltage of 5,6 V for the charging capacitor. The distance from the end of the fiber to the PV cells depends on the numerical aperture of used fiber and the dimensions of the PV cells. In Fig. 3, one can see that the light beam does not effectively cover the square cell, the loss is proportional to the ratio of the areas of the circle and the square. Therefore, the best ratio that kept the radius of the radiation circle between 0.5 and 0.707 was experimentally sought. The PV cells were based on amorphous $\mathrm{Si}(\mathrm{a}-\mathrm{Si})$.

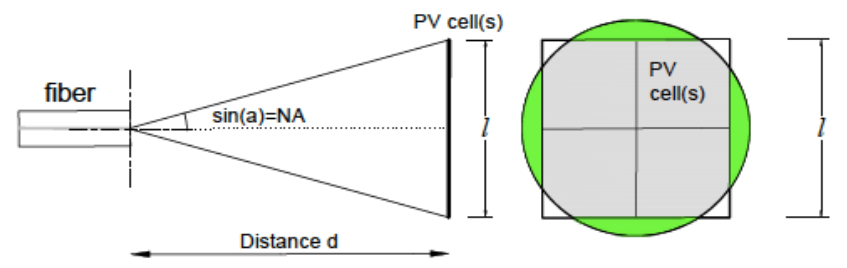

Fig. 3. Fresnel refraction to cover large dimension of PV Cell

- $\quad P V$ cell based on a-Si. Due to the limitation of the experiment to a laser wavelength at $650 \mathrm{~nm}$ (red laser) with a transmitting power of $10 \mathrm{~mW}$, the choice of PV cell is a-Si. The efficiency of a-Si ranges from 1.9 to 2.4 times less than the efficiency of c-Si [7]. However, c-Si is characterized by a drastic reduction in efficiency - when the radiation power falls below $10 \mathrm{~W} / \mathrm{m}^{2}$ it is practically unusable. The efficiency of a-Si, on the other hand, remains usable even at values of the radiation density below $0.1 \mathrm{~W} / \mathrm{m}^{2}$. For comparison, the efficiency of c-Si when the power density is less than $0.1 \mathrm{~W} / \mathrm{m}$ is below $0.2 \%$, while for a-Si it is about 5\% [7]. This phenomenon is crucial in the present 
approach; it allows us to convert a very small amount of light into electrical power. The choice of a-Si for PV cells makes the power supply system cost-effective.

\subsection{Maximum lunched power into the fiber - damage threshold}

The maximum optical power that can be inserted into the fiber must be observed through two phenomena: The first is the physical destruction of the fiber core. The second is the effect of light used to transmit energy to the information-carrying signal, in the form of nonlinear effects in the fiber due to the high power of light. The theoretical limit at which there is damage to SSM fibers (SMF-28 Ultra Fiber) is around $870 \mathrm{~mW}$, while for practical use $210 \mathrm{~mW}$ is recommended [8]. It should be noted that the power threshold depends on the effective mode area $\mathrm{A}_{\mathrm{cr}}$ that corresponds to the mode filed diameter $\left(d_{\mathrm{MDF}}\right)$ and the threshold power density $I_{\text {m }}[9]$ :

$$
P_{t h}=A_{e f f} I_{t h}=\pi\left(\frac{d_{M F D}}{2}\right)^{2} I_{t h}
$$

Depending on the specific design, values of $2.5 \mathrm{MW} / \mathrm{cm}^{2}$ are mainly used [11].

$$
d_{M D F}=2 a\left(0,65+\frac{1,619}{V^{3 / 2}}+\frac{2.879}{V^{6}}\right)
$$

\section{Experimental setup}

The experimental setup consists of the following material, and is sketched in Fig. 4:

Laser diode, wavelength $650 \mathrm{~nm}$, maximum power launched $10 \mathrm{~mW}$, optical fiber, SSM with attenuation at $650 \mathrm{~nm}$ is $6 \mathrm{~dB} / \mathrm{km}$ and length $517 \mathrm{~m}$. The distance between fiber tip and PV cells is $15,6 \mathrm{~cm}$. PV cells measure $3 \mathrm{~cm} \mathrm{x} 1 \mathrm{~cm}$ and $2.5 \mathrm{~cm}$ x $0,9 \mathrm{~cm}$, with output voltage of 5,6 V. Capacitor is electrolyte type with capacitance of $44 \mu \mathrm{F}$.

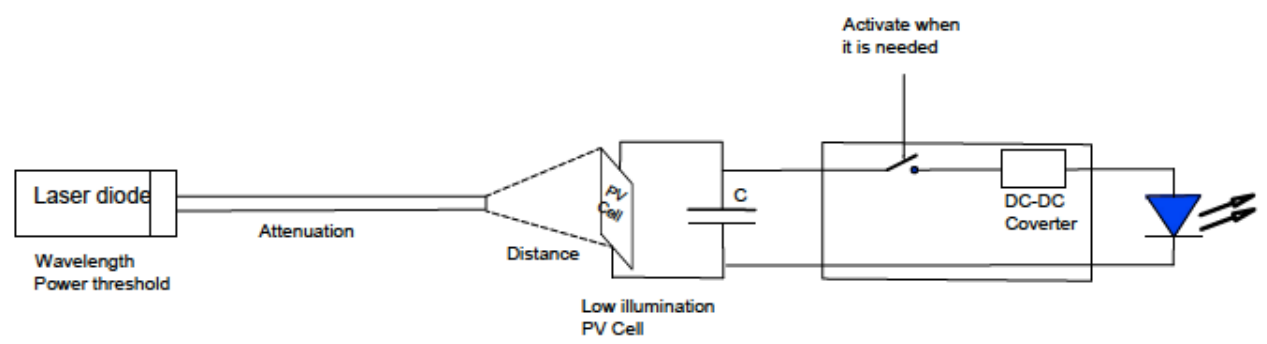

Fig. 4. Experimental setup

Waking up of the device is performed manually, Arduino and voltmeter are used to monitor voltage on the capacitor. A $220 \mathrm{Ohm}$ resistor limits the current and drops the voltage to a green LED (Fig. 5). 

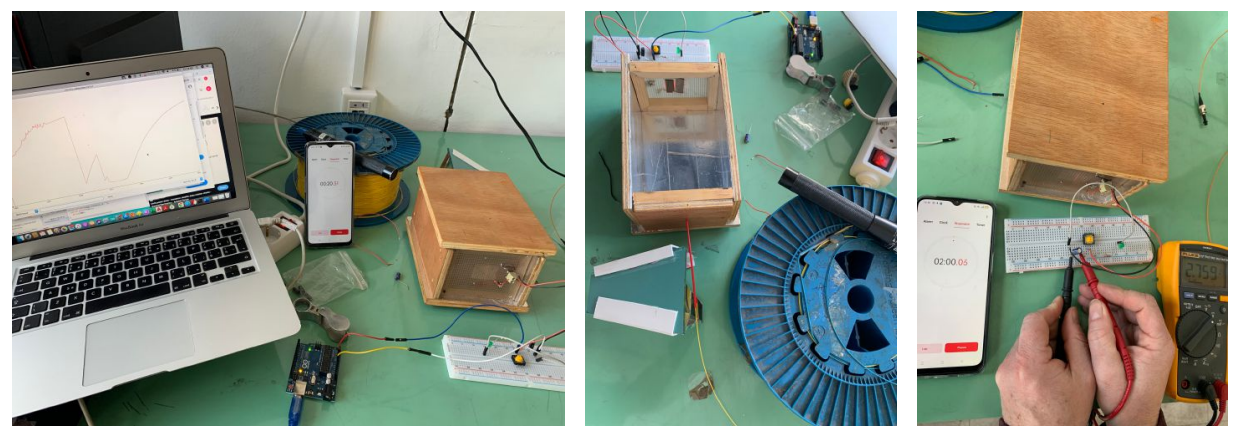

Fig. 5. Some figure from laboratory during experiment

\section{Discussion and future work}

Using inexpensive components including a-Si PV cell and a red $10 \mathrm{~mW}$ laser, in this experiment it was possible to transfer over $517 \mathrm{~m} \mathrm{SSM}$ fiber sufficient energy for an LED to emit light for $1 \mathrm{~s}$. The current which was obtained was at most around $20 \mathrm{~mA}$. Transferring more power to feed larger load would require charging a larger capacitor with more time between the awake modes. However, at a different, larger wavelength, the attenuation of the fiber decreases, the mode-field diameter increases allowing more power to be coupled to the fiber, making the system more efficient. The main reason for the low efficiency of PV cells on low radiation is the shunt resistance. In [10] authors present CIGS (Copper Indium Gallium Selenide) solar cells on low radiation and different wavelengths. For our work the wavelength of $850 \mathrm{~nm}$ could be interesting. In addition to this, a power management unit providing constant voltage at the output can further increase the efficiency. A concentrator reflecting back to PV cell the light that is not absorbed could also increase the efficiency as the refractive index of the cell is rather high.

\section{Conclusion.}

This is a report on use of inexpensive a-Si PV cells for transferring power over a long distance via a single-mode fiber for powering e.g. low-power sensors. The efficiency of a-Si at very low power densities is demonstrated making it advantageous compared to otherwise better crystalline Si.

Applications of this device are guided by its features such as low price, very long service life and the possibility of placing the device high or low temperature environments. With this in mind, the potential applications may be: Flame sensors in combination with noncombustible optical cables, to obtain information on wildfires; in mines, especially mines with flammable gases; in seismological research; in research with large long-term amounts of snow or underwater sensor network, and generally, wherever sensors are installed in a hostile environment or built into a structure after which the sensor may be difficult or impossible to access.

\section{Acknowledgements}

This project is supported by the Government of Canton Sarajevo/ Bosnia and Herzegovina: "Powering of sensors by electrical energy transferred over fiber optics (in Bosnian: "Napajanje senzora električnom energijom prenesenom preko optičkog vlakna"). 


\section{References}

1. IoT global annual revenue 2019-2030, https://www.statista.com/statistics, Published by Arne Holst, Aug 25, (2021),

2. Usmonov, Maksudjon, and Francesco Gregoretti, Design and implementation of a LoRa based wireless control for drip irrigation systems, 2017 2nd International Conference on Robotics and Automation Engineering (ICRAE). IEEE, (2017).

3. López-Cardona, J. D., et al., Remote optical powering using fiber optics in hazardous environments, Journal of Lightwave Technology 36.3 (2018): 748-754.

4. Rosolem, Joao Batista, and R. Roka, Power-over-fiber applications for telecommunications and for electric utilities, Optical fiber and wireless communications (2017): 255-278.

5. Wei, Pu, et al., Power-over-fiber system with energy management scheme for electric power industry, Optical Engineering 58.9 (2019): 096104.

6. Casaleiro, João Carlos, et al., On the Feasibility of GPON Fiber Light Energy Harvesting for the Internet of Things, i-ETC: ISEL Academic Journal of Electronics Telecommunications and Computers 4.1 (2018): 9.

7. Efika Schubert, Markus B., and Jürgen H. Werner, Flexible solar cells for clothing, Materials today 9.6 (2006): 42-50.

8. Laser-Induced Damage in Silica Optical Fibers: https://www.thorlabs.com, December (2021);

9. Todoroki, Shin-ichi, Fiber fuse propagation behavior, INTECH Open Access Publisher, (2012).

10. Swartz, Craig H., et al., Measurement of shunt resistance and conduction band offset in $\mathrm{Cu}$ (In, Ga) Se2 solar cells through joint analysis of temperature and intensity dependence of open-circuit voltage and photoluminescence, Japanese Journal of Applied Physics 59.5 (2020): 055504. 